Author(s):

Robert E. MacFarlane

Submitted to:

World Wide Web

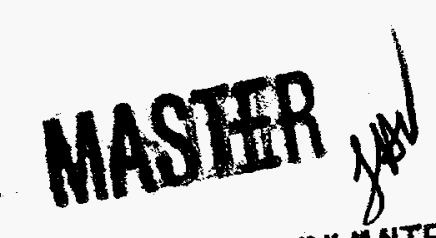

DASTRIBUTON OF THIS DOCUMENT is UNUMMTED

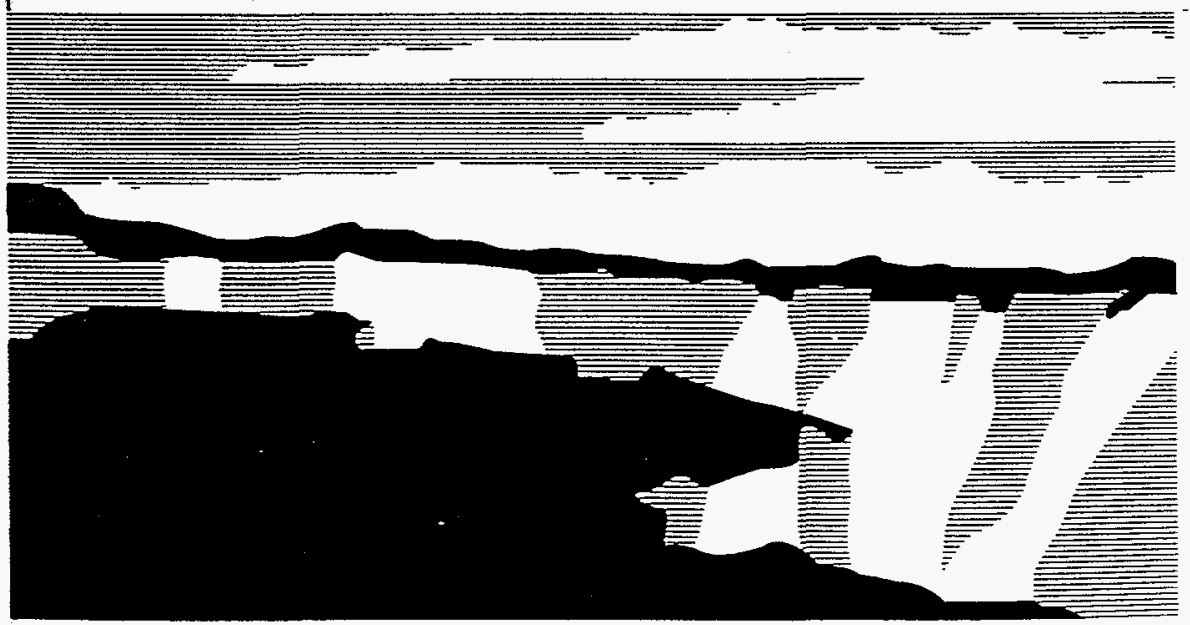

Los Alamos National Laboratory, an affirmative actionequal opportunity employer, is operated by the University of Califomia for the U.S. Department of Energy under contract W-7405-ENG-36. By acceptance of this article, the publisher recognizes that the U.S. Govemment retains a nonexclusive, royalky-free license to publish or reproduce the published form of this contribution, or to allow others to do so, for U.S. Government purposes. Los Alamos National Laboratory requests that the publisher identify this article as work periormed under the auspices of the U.S. Department of Energy. The Los Alamos National Laboratory strongly supports academic frefiom and a researcher's right 10 publish; as an institution, however, the Laboratory does not endorse the viewpoint 


\section{DISCLAIMER}

This report was prepared as an account of work sponsored by an agency of the United States Government. Neither the United States Government nor any agency thereof, nor any of their employees, makes any warranty, express or implied, or assumes any legal liability or responsibility for the accuracy, completeness, or usefulness of any information, apparatus, product, or process disclosed, or represents that its use would not infringe privately owned rights. Reference herein to any specific commercial product, process, or service by trade name, trademark, manufacturer, or otherwise does not necessarily constitute or imply its endorsement, recommendation, or favoring by the United States Government or any agency thereof. The views and opinions of authors expressed herein do not necessarily state or reflect those of the United States Government or any agency thereof. 


\section{DISCLAIMER}

Portions of this document may be illegible electronic image products. Images are produced from the best available original document. 


\title{
COLD-MODERATOR SCATTERING KERNEL METHODS
}

\author{
Robert E. MacFarlane \\ Los Alamos National Laboratory
}

\section{INTRODUCTION}

An accurate representation of the scattering of neutrons by the materials used to build cold sources at neutron scattering facilities is important for the initial design and optimization of a cold source, and for the analysis of experimental results obtained using the cold source. In practice, this requires a good representation of the physics of scattering from the material, a method to convert this into observable quantities (such as scattering cross sections), and a method to use the results in a neutron transport code (such as the MCNP Monte Carlo code). At Los Alamos, we have been developing these capabilities over the last ten years (Ref. 1, Ref. 2). The final set of cold-moderator evaluations, together with evaluations for conventional moderator materials, was released in 1994 (Ref. 3). These materials have been processed into MCNP data files using the NJOY Nuclear Data Processing System (Ref. 4). Over the course of this work, we were able to develop a new module for NJOY called LEAPR based on the LEAP+ADDELT code from the UK as modified by D. J. Picton for cold-moderator calculations. Much of the physics for methane came from Picton's work. The liquid hydrogen work was originally based on a code using the Young-Koppel approach that went through a number of hands in Europe (including Rolf Neef and Guy Robert). It was generalized and extended for LEAPR, and depends strongly on work by Keinert and Sax of the the University of Stuttgart. Thus, our collection of cold-moderator scattering kernels is truly an international effort, and we are glad to be able to return the enhanced evaluations and processing techniques to the international community.

In this paper, we will give sections on the major cold moderator materials (namely, solid methane, liquid methane, and liquid hydrogen) using each section to introduce the relevant physics for that material and to show typical results.

\section{SOLID METHANE}

Solid methane can be used to construct a cold source operating at about 22 Kelvin. The $\mathrm{CH}_{4}$ molecule is known to have fairly well defined vibrational modes at $162,190,361$, and $374 \mathrm{meV}$. When the molecules are in the solid phase (in an FCC arrangement), the spectrum of possible crystalline excitations can be represented as shown in Fig. 1 (based on measurements of Harker and Brugger). It is well known that the scattering kernel for randomly ordered microcrystals of such materials can be represented as "incoherent inelastic:"

$$
\sigma\left(E \rightarrow E^{\prime}, \mu\right)=\frac{\sigma_{b}}{2 k T} \sqrt{\frac{E^{\prime}}{E}} \mathcal{S}(\alpha, \beta),
$$

where $E$ and $E^{\prime}$ are the incident and secondary neutron energies in the laboratory system, $\mu$ is the cosine of the scattering angle in the laboratory, $\sigma_{b}$ is the characteristic bound scattering cross section for the material, $k T$ is the thermal energy in $\mathrm{eV}$, and $\mathcal{S}$ is the 


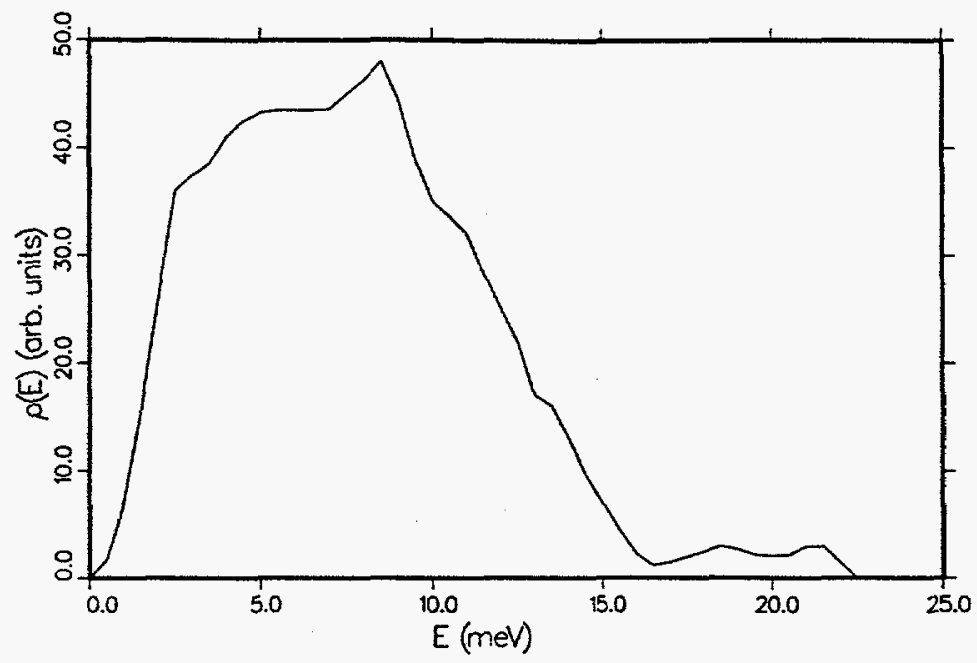

Figure 1: The Harker-Brugger frequency spectrum used for solid methane. Note the quadratic shape at low energies.

asymmetric form of the scattering law. The scattering law depends on only two variables, the momentum transfer

$$
\alpha=\frac{E^{\prime}+E-2 \sqrt{E^{\prime} E} \mu}{A k T},
$$

where $A$ is the ratio of the scatterer mass to the neutron mass, and the energy transfer

$$
\beta=\frac{E^{\prime}-E}{k T} .
$$

In the LEAPR module of NJOY, the scattering law for solid-like excitations is computed from the frequency distribution $\rho(\beta)$ using the "phonon expansion." The details will be found in Ref. 3; we will just give the final result here.

$$
\mathcal{S}_{s}(\alpha, \beta)=\mathrm{e}^{-\alpha \lambda_{s}} \sum_{n=0}^{\infty} \frac{1}{n !}\left[\alpha \lambda_{s}\right]^{n} \mathcal{T}_{n}(\beta),
$$

where

$$
\begin{gathered}
\mathcal{T}_{0}(\beta)=\delta(\beta) \\
\mathcal{T}_{1}(\beta)=\frac{P_{s}(\beta) \mathrm{e}^{-\beta / 2}}{\lambda_{s}}
\end{gathered}
$$

and

$$
\mathcal{T}_{n}(\beta)=\int_{-\infty}^{\infty} \mathcal{T}_{1}\left(\beta^{\prime}\right) \mathcal{T}_{n-1}\left(\beta-\beta^{\prime}\right) d \beta^{\prime}
$$

In these equations, $\lambda$ is the Debye-Waller coefficient for the solid-like modes

$$
\lambda_{s}=\int_{-\infty}^{\infty} P_{s}(\beta) \mathrm{e}^{-\beta / 2} d \beta,
$$


the frequency spectrum enters through

$$
P_{s}(\beta)=\frac{\rho(\beta)}{2 \beta \sinh (\beta / 2)},
$$

and $\mathcal{T}_{n}$ is normalized when integrated over all beta. We simply compute the $\mathcal{T}_{n}(-\beta)$ function on the input beta grid for $\mathrm{n}$ up to some specified maximum value, typically 100 . It is then easy to compute the smooth part of $\mathcal{S}(\alpha,-\beta)$ for any sufficiently small value of alpha. The corresponding values of $\mathcal{S}(\alpha, \beta)$ can then be obtained by multiplying by $\exp (-\beta)$. The delta function from the "zero-phonon" term is carried forward separately.

This result can be extended to larger alpha and beta values by using the "short collision time" approximation

$$
\mathcal{S}_{s}(\alpha,-\beta)=\frac{1}{\sqrt{4 \pi w_{s} \alpha \bar{T}_{s} / T}} \exp \left[-\frac{\left(w_{s} \alpha-\beta\right)^{2}}{w_{s} \alpha \bar{T}_{s} / T}\right],
$$

and

$$
\mathcal{S}_{s}(\alpha, \beta)=\mathrm{e}^{-\beta} \mathcal{S}_{s}(\alpha,-\beta)
$$

where $\beta$ is positive, and where the effective temperature is given by

$$
\bar{T}_{s}=\frac{T}{2 w_{s}} \int_{-\infty}^{\infty} \beta^{2} P_{s}(\beta) \mathrm{e}^{-\beta} d \beta
$$

In these formulas, $w_{s}$ is the weight for the solid-type modes.

Once the solid part is available, the discrete excitations can be added using a convolution method. Skipping details that can be found in the reference, the result has the form

$$
\mathcal{S}(\alpha, \beta)=\sum_{k} W_{k}(\alpha) \mathcal{S}_{s}\left(\alpha, \beta-\beta_{k}\right),
$$

where the $\beta_{k}$ and the associated weights $W_{k}$ are generated using a recursive procedure. The Debye-Waller $\lambda$ for the combined modes is computed using

$$
\lambda=\lambda_{s}+\sum_{i=1}^{N} \lambda_{i}
$$

The effective temperature for the combined modes is given by

$$
\bar{T}_{s}=w_{t} T+w_{s} \bar{T}_{s}+\sum_{i=1}^{N} w_{i} \frac{\beta_{i}}{2} \operatorname{coth}\left(\frac{\beta_{i}}{2}\right) T .
$$

It is important to remember to include the effects of the zero-phonon term from Eq. 4 $\exp (-\alpha \lambda) \delta(\beta)$. The code does this by adding in triangular peaks with the proper areas and with their apexes at the beta value closest to the $\beta_{k}$ values. The peak at $\beta=0$ is not put in as a sharp triangle; instead, it is handled as "incoherent elastic" scattering in order to take full advantage of the analytic properties of $\delta(\beta)$. The scattering function that combines these various effects is shown in Fig. 2.

The incoherent inelastic term arising from the zero-phonon term is given by

$$
\mathcal{S}_{\text {iel }}(\alpha, \beta)=\mathrm{e}^{-\alpha \lambda} \delta(\beta)
$$

and the corresponding differential and integrated scattering cross sections are

$$
\sigma(E, \mu)=\frac{\sigma_{b}}{2} \mathrm{e}^{-2 W E(1-\mu)}
$$




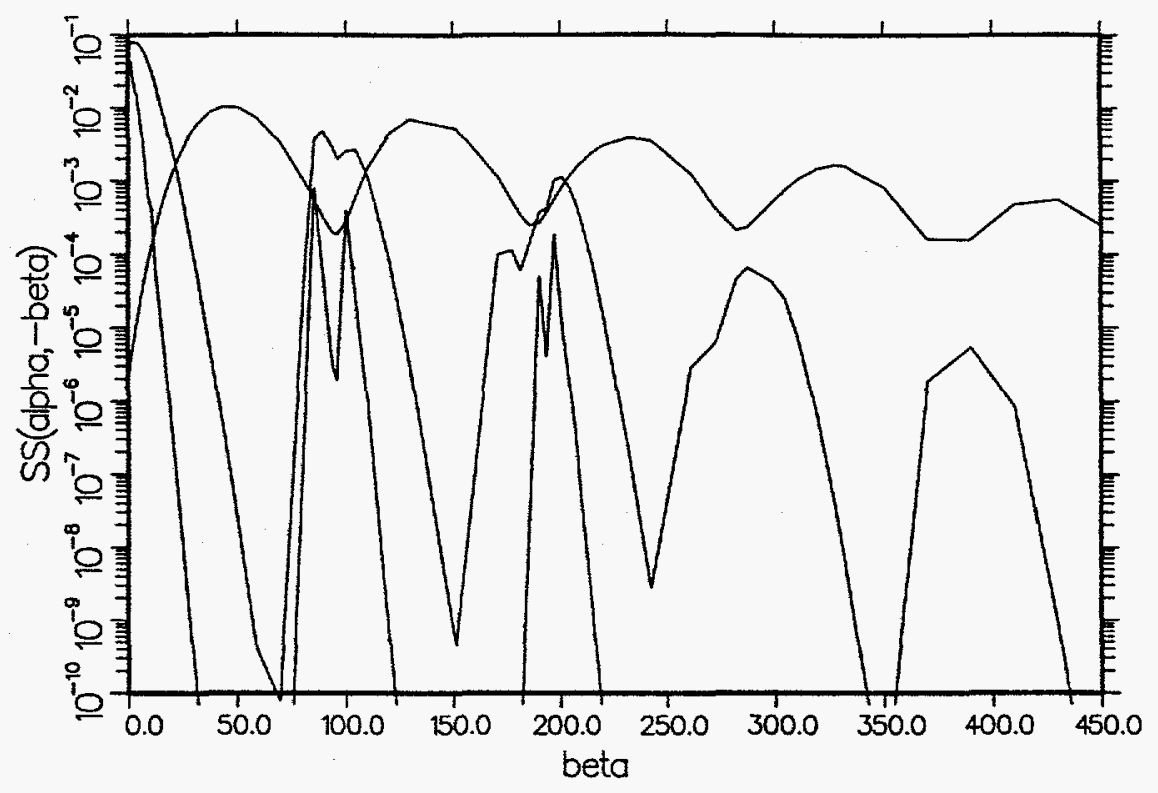

Figure 2: $\mathcal{S}(\alpha,-\beta)$ for solid methane shown as a function of $\beta$ for the $\alpha$ values 0.986 (lowest), 16.94, and 151.45 (highest). The four discrete levels show up as sharp triangles in the lowest curve.

and

$$
\sigma(E)=\frac{\sigma_{b}}{2}\left\{\frac{1-\mathrm{e}^{-4 W E}}{2 W E}\right\} .
$$

In these equations, the Debye-Waller coefficient is given by

$$
W=\frac{\lambda}{A k T}
$$

The incoherent inelastic and incoherent elastic cross sections for solid methane are shown in Fig. 3. Some examples of the spectrum of outgoing neutrons corresponding to different incident neutron energies are shown in Fig. 3.

\section{LIQUID METHANE}

Liquid methane can be used to build cold sources at about 90 Kelvin. Neutron scattering experiments show surprisingly little change when a material changes from a solid to a liquid. It is almost as if a liquid consists of little chunks of solid material moving through each other by a diffusive mechanism. Some of the structure in the frequency distribution might be expected to smooth out, and the sharp delta functions associated with the zero-phonon term in the solid tend to broaden out into quasi-elastic peaks. Fig. 4 shows the solidtype frequency spectrum that we used for liquid methane (it shows the normal quadratic dependence at small beta). This particular function came from Picton based on work by Agrawal and Yip and modified to be used with the ENDF representation for diffusion. More details are available in Ref. 4. 

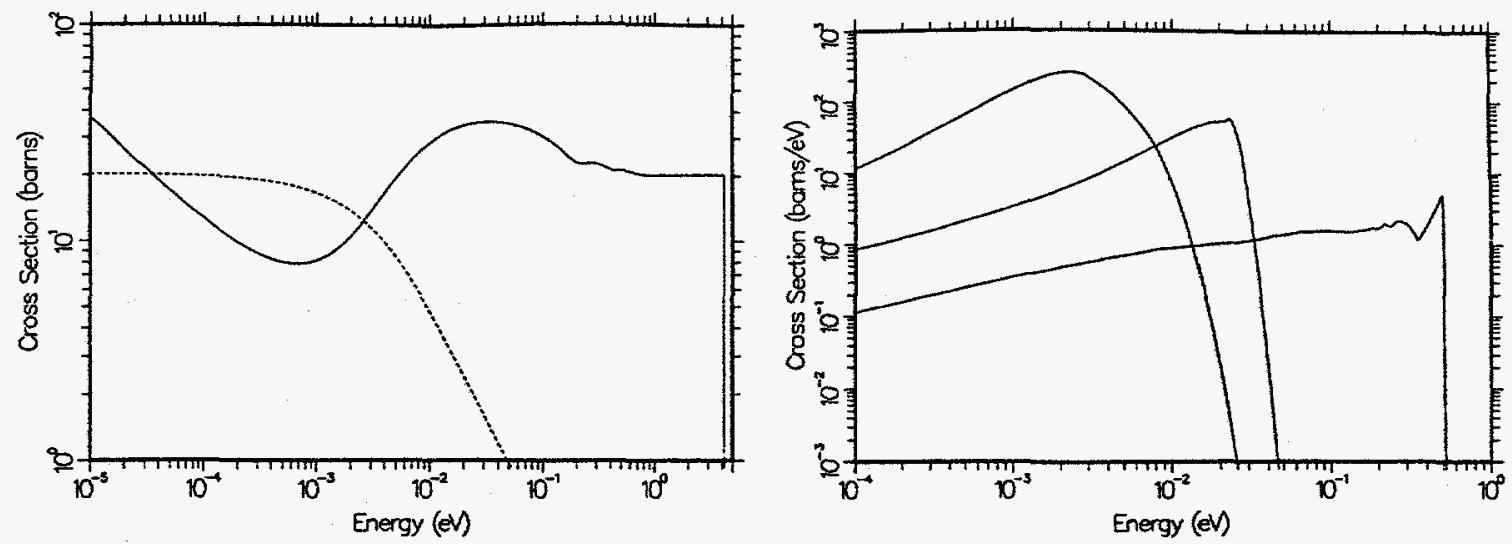

Figure 3: Left: Inelastic (solid) and incoherent elastic (dashed) cross sections for solid methane. The small bumps starting at about $0.2 \mathrm{eV}$ are due to the discrete levels at .162, $.190, .361$, and $.374 \mathrm{eV}$. Right: Neutron spectra $\sigma\left(E \rightarrow E^{\prime}\right)$ for solid methane shown as functions of outgoing neutron energy $E^{\prime}$ for $E=0.0001, .0253$, and $.503 \mathrm{eV}$.

The simple ENDF diffusion mode uses the following form for the scattering law:

$$
\begin{aligned}
\mathcal{S}_{t}(\alpha, \beta) & =\frac{2 c w_{t} \alpha}{\pi} \exp \left[2 c^{2} w_{t} \alpha-\beta / 2\right] \\
& \times \frac{\sqrt{c^{2}+.25}}{\sqrt{\beta^{2}+4 c^{2} w_{t}^{2} \alpha^{2}}} K_{1}\left\{\sqrt{c^{2}+.25} \sqrt{\beta^{2}+4 c^{2} w_{t}^{2} \alpha^{2}}\right\}
\end{aligned}
$$

and

$$
\rho(\beta)=w_{t} \frac{4 c}{\pi \beta} \sqrt{c^{2}+.25} \sinh (\beta / 2) K_{1}\left\{\sqrt{c^{2}+.25} \beta\right\} .
$$

Here $K_{1}$ is modified Bessel function of the second kind, and the translational weight $w_{t}$ and the diffusion constant $c$ are provided as inputs. We chose them to give a reasonable representation of the full width at half maximum of the quasi-elastic peak as observed in neutron scattering experiments. When this diffusion law is combined with the solid-type modes by convolution, the result has the form

$$
\mathcal{S}(\alpha, \beta)=\mathcal{S}_{t}(\alpha, \beta) \mathrm{e}^{-\alpha \lambda_{s}}+\int_{-\infty}^{\infty} \mathcal{S}_{t}\left(\alpha, \beta^{\prime}\right) \mathcal{S}_{s}\left(\alpha, \beta-\beta^{\prime}\right) d \beta^{\prime}
$$

where the first term comes from the zero-phonon delta function. The effective temperature for this combination of modes is given by

$$
\bar{T}_{s}=\frac{w_{t} T+w_{s} \bar{T}_{s}}{w_{t}+w_{s}}
$$

Some typical $\mathcal{S}(\alpha, \beta)$ curves for liquid methane are shown in Fig. 4. The sharp difference in behavior of the $\beta=0$ curve as alpha approaches zero is characteristic of diffusion. The computed cross section is compared to experimental data in Fig. 5, which also shows neutron spectra for various incident energies. 

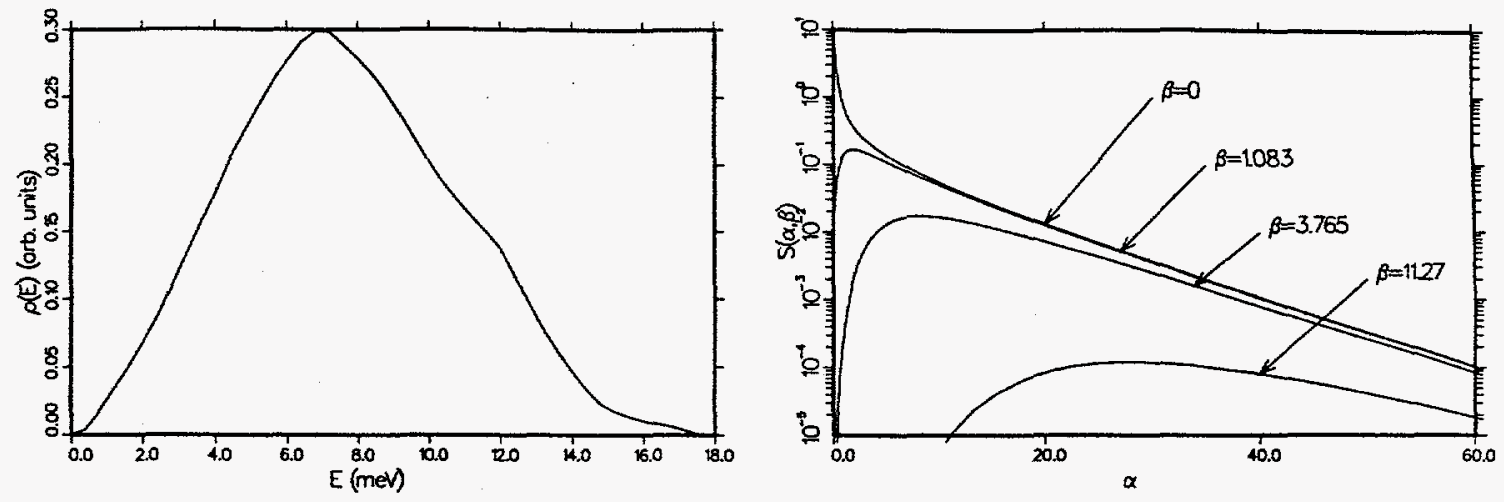

Figure 4: Effective frequency spectrum for methane including both translational and rotational modes, but not including diffusive modes (left), and $\mathcal{S}(\alpha, \beta)$ curves for liquid methane (right). Note the diffusive behavior at low $\alpha$ and $\beta$.

\section{LIQUID HYDROGEN AND DEUTERIUM}

Liquid hydrogen and deuterium have been used to build a number of cold sources that operate in the region of 20 Kelvin. These materials violate the assumption that spins are distributed randomly that allowed us to use the incoherent approximation for solid and liquid methane. An explicitely quantum-mechanical formulation is required to take account of the correlations between the spins of the two atoms in the same molecule. This problem was considered by Young and Koppel, and with some changes in notation,

$$
\begin{aligned}
\mathcal{S}_{\text {para }}(\alpha, \beta) & =\sum_{J=0,2,4, \ldots} P_{J} \\
& \times \frac{4 \pi}{\sigma_{b}}\left[A_{\text {para }} \sum_{J^{\prime}=0,2,4, \ldots}+B_{\text {para }} \sum_{J^{\prime}=1,3,5, \ldots}\right]\left(2 J^{\prime}+1\right) \\
& \times \mathcal{S}_{f}\left(w \alpha, \beta+\beta_{J J^{\prime}}\right) \\
& \times \sum_{\ell=\left|J^{\prime}-J\right|}^{J^{\prime}+J} 4 j_{\ell}^{2}(y) C^{2}\left(J J^{\prime} \ell ; 00\right),
\end{aligned}
$$

and

$$
\begin{aligned}
\mathcal{S}_{\text {ortho }}(\alpha, \beta) & =\sum_{J=1,3,5, \ldots} P_{J} \\
& \times \frac{4 \pi}{\sigma_{b}}\left[A_{\text {ortho }} \sum_{J^{\prime}=0,2,4, \ldots}+B_{\text {ortho }} \sum_{J^{\prime}=1,3,5, \ldots}\right]\left(2 J^{\prime}+1\right) \\
& \times \mathcal{S}_{f}\left(w \alpha, \beta+\beta_{J J^{\prime}}\right) \\
& \times \sum_{\ell=\left|J^{\prime}-J\right|}^{J^{\prime}+J} 4 j_{\ell}^{2}(y) C^{2}\left(J J^{\prime} \ell ; 00\right) .
\end{aligned}
$$

The coefficents for the even and odd sums are given in the following table: 

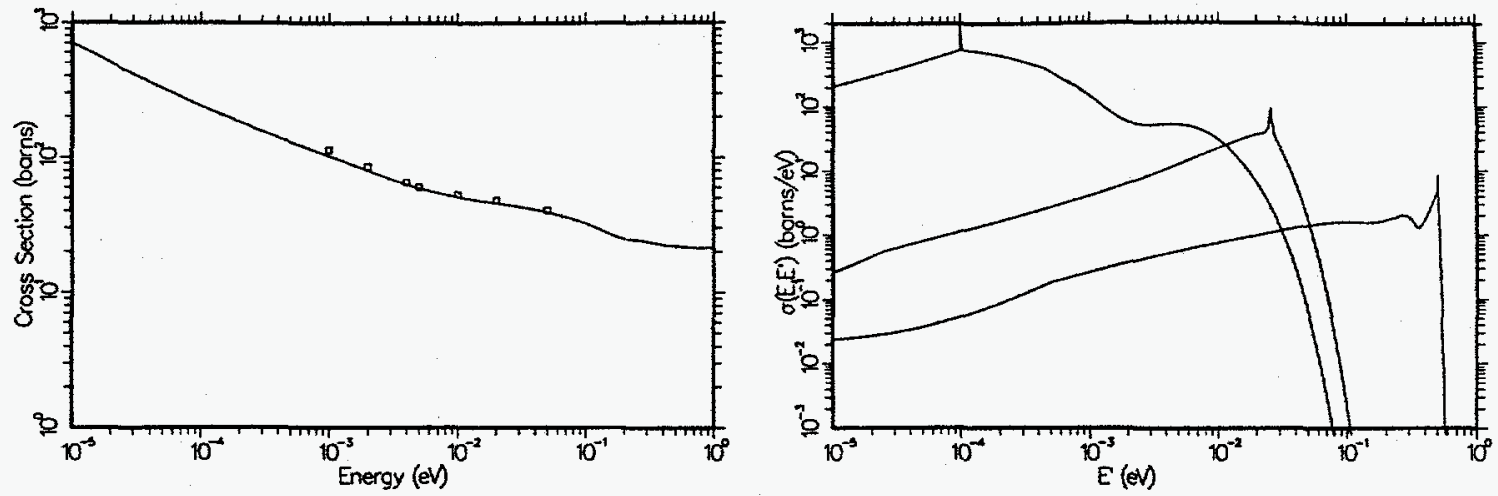

Figure 5: The computed cross section for liquid methane at $100 \mathrm{~K}$ (solid) is compared to experimental data (squares) by Whittemore and by Rogalska as quoted by Agrawal and Yip (left) and neutron spectra $\sigma\left(E \rightarrow E^{\prime}\right)$ are shown for $E=.0001, .0253$, and $.503 \mathrm{meV}$ (right). Note the sharp quasi-elastic peak that results from the diffusive term in the theory used here.

\begin{tabular}{lcc} 
Type & $A($ even $)$ & $B$ (odd) \\
\hline H para & $a_{c}^{2}$ & $a_{i}^{2}$ \\
H ortho & $a_{c}^{2} / 3$ & $a_{c}^{2}+2 a_{i}^{2} / 3$ \\
D para & $3 a_{i}^{2} / 4$ & $a_{c}^{2}+a_{i}^{2} / 4$ \\
D ortho & $a_{c}^{2}+5 a_{i}^{2} / 8$ & $3 a_{i}^{2} / 8$ \\
\hline
\end{tabular}

Here $a_{c}$ and $a_{i}$ are the coherent and incoherent scattering lengths (note that the characteristic bound cross section $\left.\sigma_{b}=4 \pi\left[a_{c}^{2}+a_{i}^{2}\right]\right), P_{J}$ is the statistical weight factor, $\beta_{J J^{\prime}}=\left(E_{J}^{\prime}-E_{J}\right) / k T$ is the energy transfer for a rotational transition, $j_{\ell}(x)$ is a spherical Bessel function of order $\ell$, and $C\left(J J^{\prime} \ell ; 00\right)$ is a Clebsch-Gordan coefficient. The parameter $y$ is given by $\kappa a / 2=(a / 2) \sqrt{M k T \alpha}$, where $a$ is the interatomic distance in the molecule, and $M$ is the molecular mass. The translational weight $w$ is $1 / 2$ for $\mathrm{H}_{2}$ and $1 / 4$ for $\mathrm{D}_{2}$. The sums over $J^{\prime}$ are treated as operators into order to keep the notation compact.

Young and Koppel assumed that the molecular translations were free, so the equations contain

$$
\mathcal{S}_{f}(\alpha,-\beta)=\frac{1}{\sqrt{4 \pi \alpha}} \exp \left[-\frac{(\alpha-\beta)^{2}}{4 \alpha}\right]
$$

and

$$
\mathcal{S}_{f}(\alpha, \beta)=\mathrm{e}^{-\beta} \mathcal{S}_{f}(\alpha,-\beta),
$$

the free-atom scattering function (with $\beta$ positive). Note that $\alpha$ is multiplied by a translational weight of 0.5 or 0.25 when this equation is used in order to make the formula apply to a molecule with mass ratio 2 or 4 , respectively.

However, in a liquid, there are two additional effects to be considered: interference between the neutron waves scattered from different molecules, and the fact that the recoil of the hydrogen molecule is not really free. We treat the latter effect by picturing a hydrogen molecule bound in a cluster of about 20 molecules and undergoing vibrations or hindered rotations similar to those of a hydrogen molecule in a solid (Keinert and Sax). These clumps then diffuse through the liquid (hindered translations) according to the simple ENDF formulation of diffusion (this part was added at Los Alamos). 

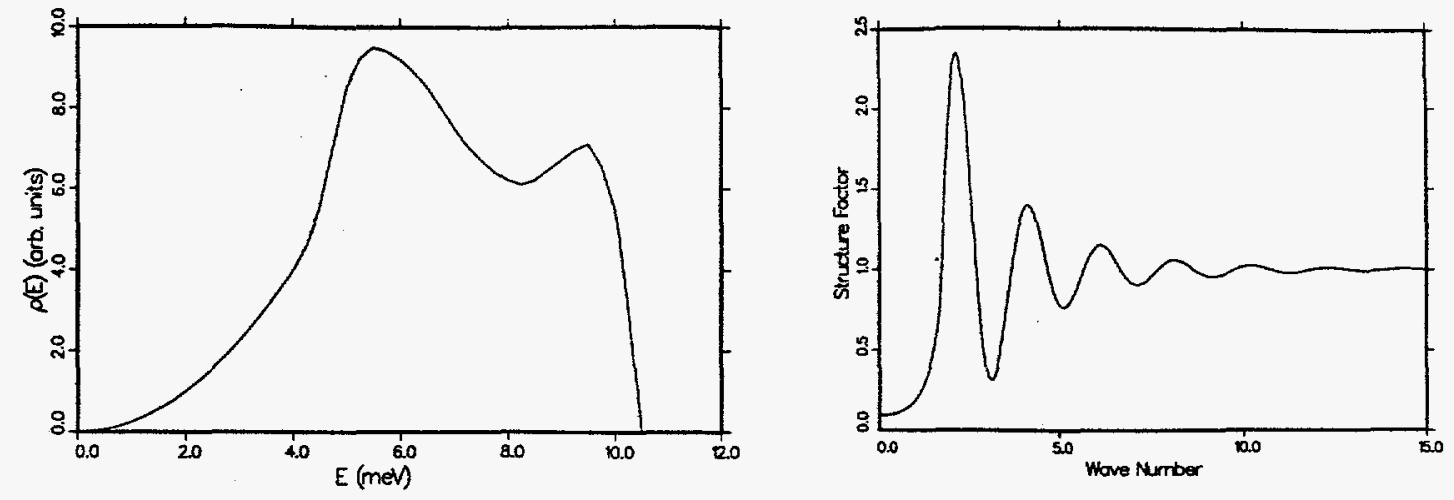

Figure 6: The Keinert-Sax frequency distribution for the effective translational modes (left) and static structure factor $S(\kappa)$ for liquid hydrogen.
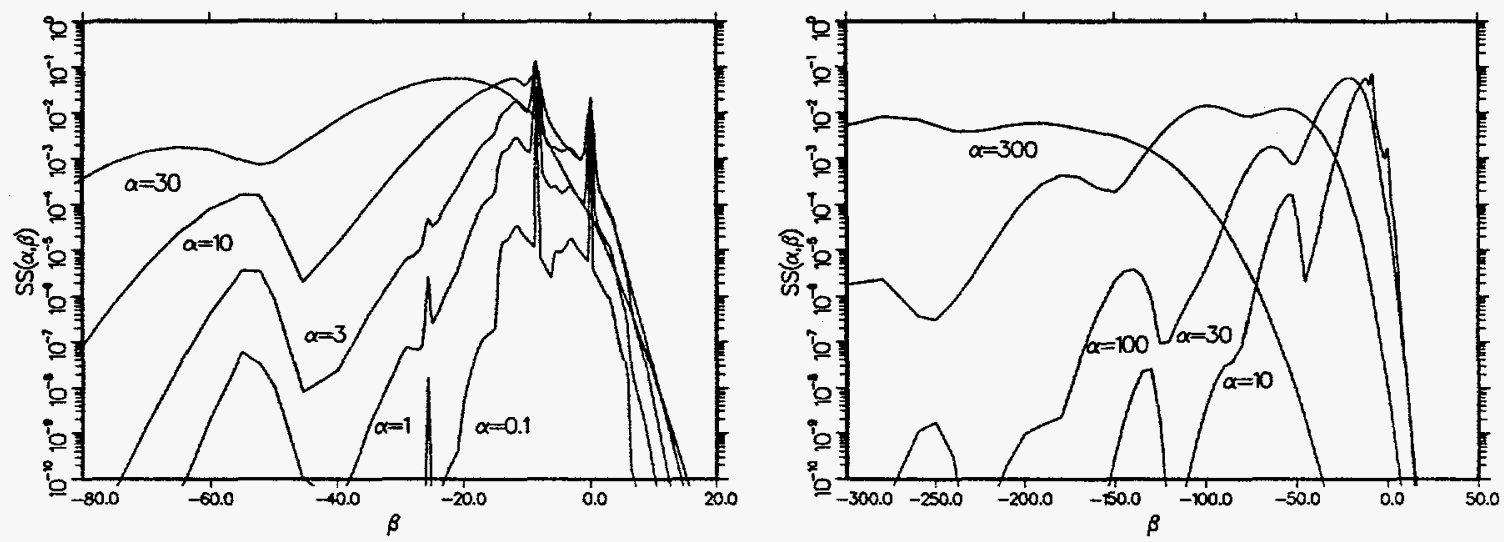

Figure 7: Script-S for liquid para hydrogen at $20 \mathrm{~K}$ is shown as a function of $\beta$ for several $\alpha$ values. The left side concentrates on the middle range of $\alpha$ and $\beta$ values, and the right shows the large values.

Coherent interference between waves scattered from two different molecules comes about when there is a correlation between their positions. This kind of spacing correlation is described by the "static structure factor" $S(\kappa)$. This quantity can be used in an approximation due to Vineyard to get

$$
\frac{d^{2} \sigma}{d \Omega d \epsilon}=\frac{d^{2} \sigma_{\mathrm{coh}}}{d \Omega d \epsilon} S(\kappa)+\frac{d^{2} \sigma_{\mathrm{incoh}}}{d \Omega d \epsilon} .
$$

This is equivalent to using Eqs. 24 and 25 with $a_{c}^{2}$ replaced by $S(\kappa) a_{c}^{2}$ in the calculation of the coefficients $A$ and $B$.

The two main components of the calculation for liquid hydrogen are shown in Fig. 6; namely, the Keinert-Sax frequency distribution $\rho(E)$ and the static structure factor $S(\kappa)$. The results for the scattering law are very complex, and they are difficult to represent well over the large ranges of alpha and beta required by the low temperatures. Some examples are shown in Figs. 7 and 8.

The integrated cross sections for the ortho and para phases as obtained from these 


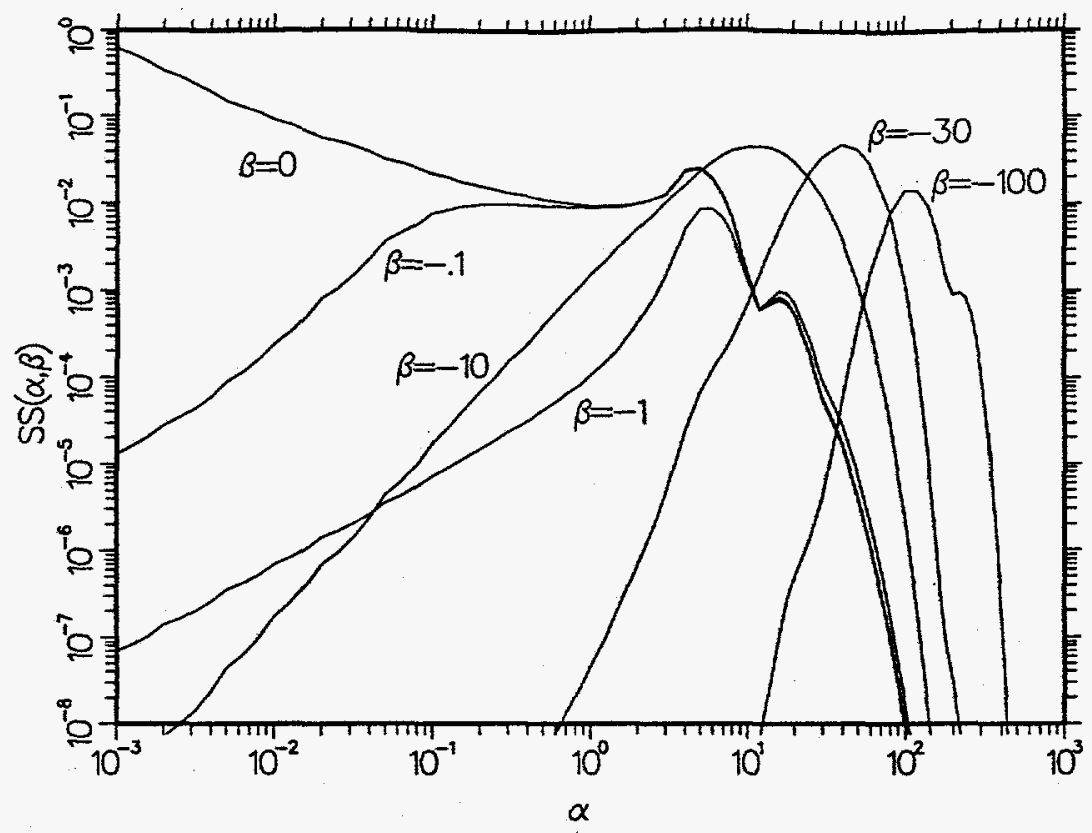

Figure 8: Script-S for liquid para hydrogen at $20 \mathrm{~K}$ is shown as a function of $\alpha$ for several $\beta$ values corresponding to downscatter.

scattering laws are compared to experimental data in Fig. 9. The sharp drop in the para cross section below $.05 \mathrm{eV}$ is due to the intermolecular spin correlation. The second drop below $3 \mathrm{meV}$ is due to the intramolecular correlation. Typical neutron emission spectra are shown in Fig. 10.

The integrated cross sections for liquid deuterium are shown in Fig. 11. The difference between the ortho and para phases is not as sharp as for hydrogen.

\section{MONTE CARLO APPLICATIONS}

We process these scattering kernel evaluations into libraries for use in the MCNP Monte Carlo code. While passing through the THERMR module of NJOY, the angular distributions are converted into NA equally probable cosine values. In the ACER module, the representation is simplified further into NE discrete scattered energies using a probability pattern of the form 1-4-10-10...10-4-1 in order to put a few more samples out onto the wings of the distribution. Therefore, MCNP can sample from the kernel by producing these $\mathrm{NA}^{*} \mathrm{NE}$ discrete events. This form is very economical, and it is easy to interpolate to intermediate incident energies. However, it is only suitable if each neutron undergoes several collisions before being detected. In some situations with thin regions, the discrete nature of the representation can lead to artifacts in the results.

\section{CONCLUSIONS}

These evaluations for the predominant cold-moderator materials reflect our current knowledge of the physics of the materials fairly well, and they lend themselves well to 


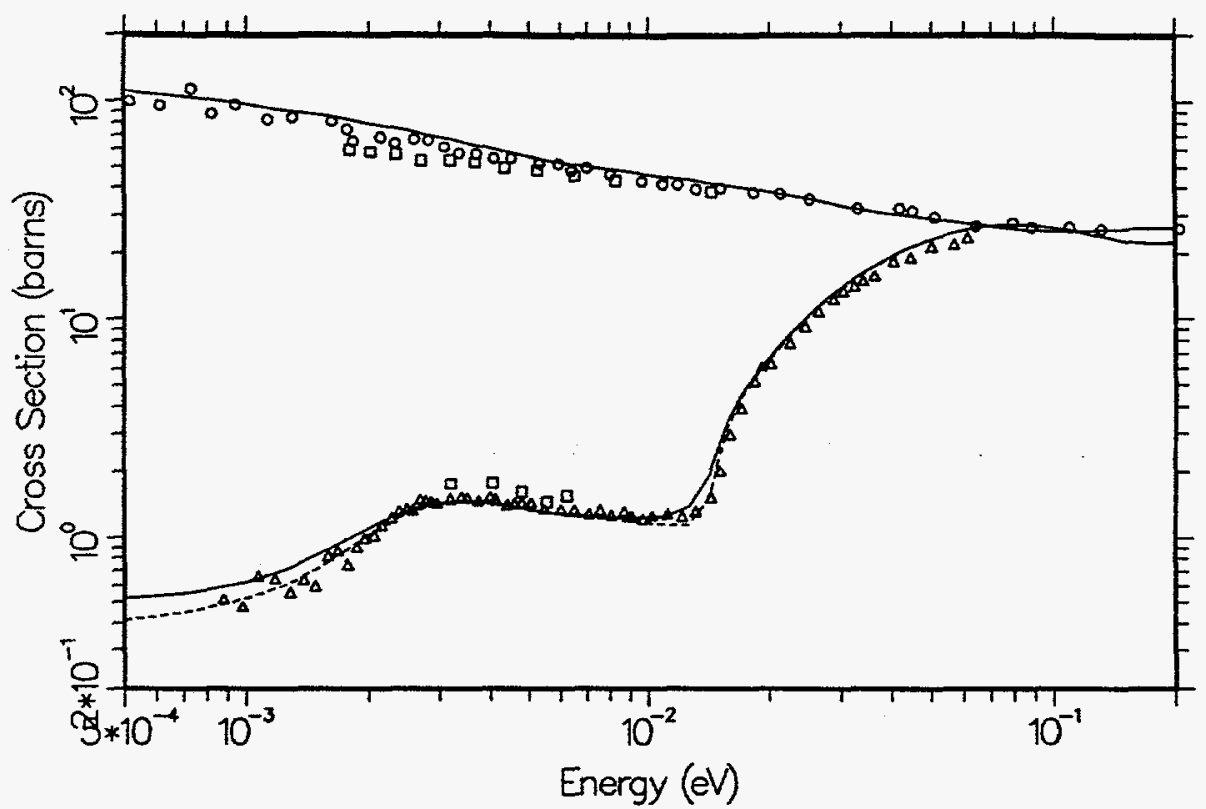

Figure 9: The cross sections for liquid ortho hydrogen (upper curve) and liquid para hydrogen (lower curve) are compared with experimental data due to Squires (gas) at 20K (squares), Whittemore at $20 \mathrm{~K}$ (circles), and Seiffert at $14 \mathrm{~K}$ (triangles). The solid curves are at $20 \mathrm{~K}$, and the dashed curve is at $14 \mathrm{~K}$. The sharp drop in the para cross section below $0.05 \mathrm{eV}$ is due due to spin coherence, and the second drop below $.003 \mathrm{eV}$ is due to intermolecular interference.

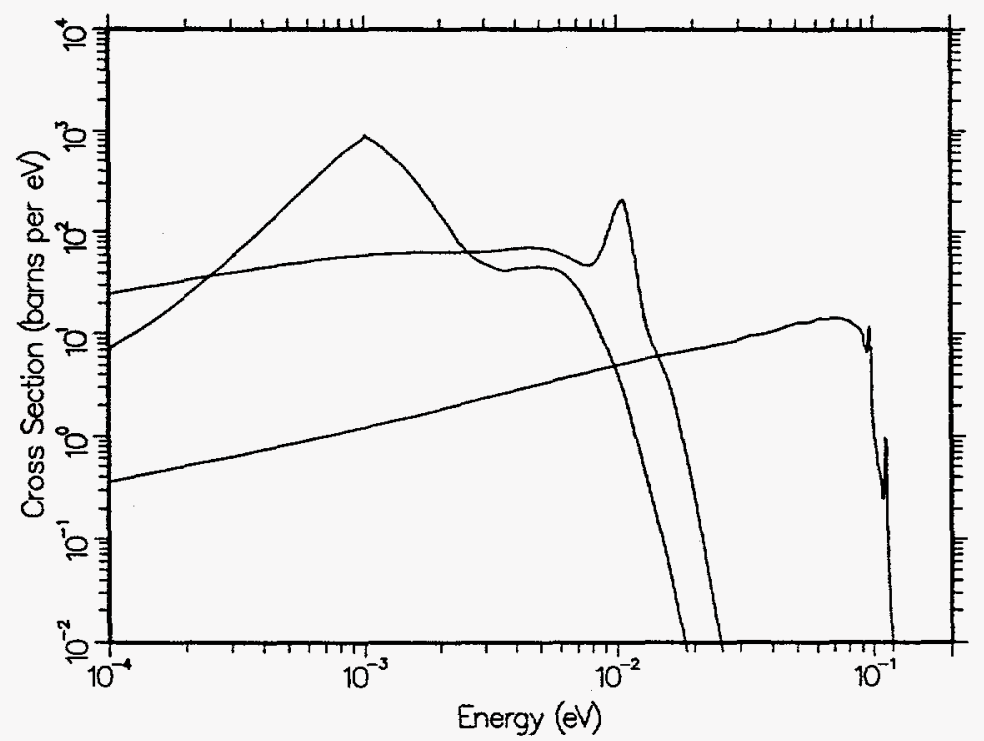

Figure 10: The spectra $\sigma\left(E \rightarrow E^{\prime}\right)$ for liquid para hydrogen are shown for $E=.001, .0106$, and $.112 \mathrm{eV}$. 


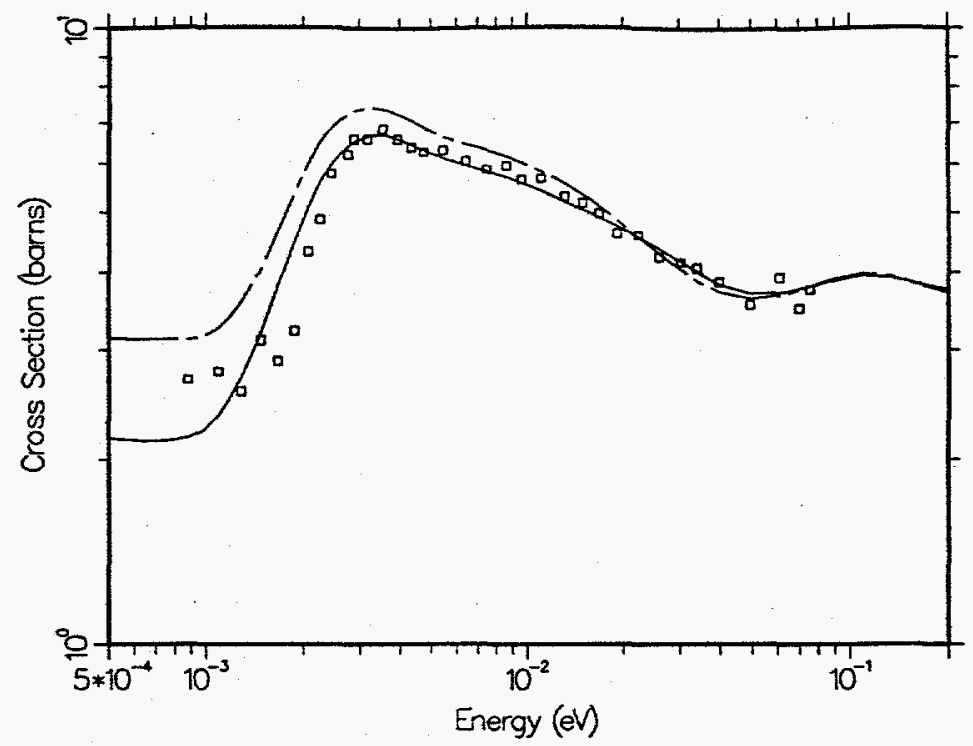

Figure 11: The cross sections for liquid para deuterium (solid curve) and liquid ortho deuterium (chain-dash curve) at $19 \mathrm{~K}$ are compared with experimental data of Seiffert for an equilibrium ortho-para mixture at $19 \mathrm{~K}$. The drop in the cross sections below $.003 \mathrm{eV}$ is due to intermolecular interference.

processing into libraries for use in detailed neutron transport calculations. Additional details are available in Ref. 4 , and also online through $h t t p: / / t 2 . l a n l . g o v$. Some of these files have been made available informally, but a packaged and validated set will be released by the Los Alamos Monte Carlo Group sometime next year.

\section{REFERENCES}

1. Robert E. MacFarlane, "Cold Moderator Scattering Kernels," in Advanced Neutron Sources 1988, Proceedings of the 10th Meeting of the International Collaboration on Advanced Neutron Sources (ICANS-X) held at Los Alamos, 3-7 October 1988, Institute of Physics Conferences Series Number 97 (Institute of Phyiscs, Bristol and New York, 1988), p. 157.

2. R. E. MacFarlane, "Cold Moderator Scattering Kernels," presented at the International Workshop on Cold Neutron Sources, March 5-8, 1990, Los Alamos, New Mexico, Los Alamos National Laboratory report LA-12146-C (August 1991).

3. R. E. MacFarlane, "New Thermal Neutron Scattering Files for ENDF/B-VI Release 2," Los Alamos National Laboratory report LA-12639-MS (ENDF 356) (March 1994).

4. R. E. MacFarlane and D. W. Muir, "The NJOY Nuclear Data Processing System, Version 91," Los Alamos National Laboratory report LA-12740-MS (October 1994): 